\title{
lonized gas in the Scutum spiral arm as traced in [N II] and [C II]
}

\author{
W. D. Langer ${ }^{1}$, T. Velusamy ${ }^{1}$, P. F. Goldsmith ${ }^{1}$, J. L. Pineda ${ }^{1}$, E. T. Chambers ${ }^{2}$, G. Sandell ${ }^{2}$, \\ C. Risacher ${ }^{3}$, and K. Jacobs ${ }^{4}$ \\ 1 Jet Propulsion Laboratory, California Institute of Technology, 4800 Oak Grove Drive, Pasadena, CA 91109, USA \\ e-mail: William.Langer@jpl.nasa.gov \\ 2 SOFIA-USRA, NASA Ames Research Center, MS 232-12, Moffett Field, CA 94035-0001, USA \\ 3 Max-Planck-Institut für Radioastronomie, Auf dem Hügel 69, 53121 Bonn, Germany \\ 4 I. Physikalisches Institut der Universität zu Köln, Zülpicher Strasse 77, 50937 Köln, Germany
}

Received 18 May 2017 / Accepted 26 July 2017

\begin{abstract}
Context. The warm ionized medium (WIM) occupies a significant fraction of the Galactic disk. Determining the WIM properties at the leading edge of spiral arms is important for understanding its dynamics and cloud formation.

Aims. We derive the properties of the WIM at the inner edge of the Scutum arm tangency, which is a unique location in which to disentangle the WIM from other components, using the ionized gas tracers $\mathrm{C}^{+}$and $\mathrm{N}^{+}$.

Methods. We use high spectral resolution [C II] $158 \mu \mathrm{m}$ and [N II] $205 \mu \mathrm{m}$ fine structure line observations taken with the upGREAT and GREAT instruments, respectively, on SOFIA, along with auxiliary $\mathrm{H} \mathrm{I}$ and ${ }^{13} \mathrm{CO}$ observations. The observations consist of samples in and out of the Galactic plane along 18 lines of sight (LOS) between longitude $30^{\circ}$ and $32^{\circ}$.

Results. We detect strong [N II] emission throughout the Scutum tangency. At $V_{\mathrm{LSR}}=110$ to $125 \mathrm{~km} \mathrm{~s}^{-1}$ where there is little, if any, ${ }^{13} \mathrm{CO}$, we are able to disentangle the $[\mathrm{N} \mathrm{II}]$ and $[\mathrm{C} \mathrm{II}]$ emission that arises from the WIM at the arm's inner edge. We find an average electron density $\sim 0.9 \mathrm{~cm}^{-3}$ in the plane, and $\sim 0.4 \mathrm{~cm}^{-3}$ just above the plane. The [N II] emission decreases exponentially with latitude with a scale height $\sim 55 \mathrm{pc}$. For $V_{\mathrm{LSR}}<110 \mathrm{~km} \mathrm{~s}^{-1}$ there is [N II] emission tracing highly ionized gas throughout the arm's molecular layer. This ionized gas has a high density, $n(\mathrm{e}) \sim 30 \mathrm{~cm}^{-3}$, and a few percent filling factor. We also find evidence for [C II] absorption by foreground gas.

Conclusions. [N II] and [C II] observations at the Scutum arm tangency reveal a highly ionized gas with average electron density about 10 to 20 times those of the interarm WIM, and is best explained by a model in which the interarm WIM is compressed as it falls into the potential well of the arm. The widespread distribution of [N II] in the molecular layers shows that high density ionized gas is distributed throughout the Scutum arm. The electron densities derived from [N II] for these molecular cloud regions are $\sim 30 \mathrm{~cm}^{-3}$, and probably arise in the ionized boundary layers of clouds. The [N II] detected in the molecular portion of the spiral arm arises from several cloud components with a combined total depth $\sim 8 \mathrm{pc}$. This [N II] emission most likely arises from ionized boundary layers, probably the result of the shock compression of the WIM as it impacts the arm's neutral gas, as well as from extended H II regions.
\end{abstract}

Key words. ISM: clouds - ISM: structure - photon-dominated region (PDR) - infrared: ISM

\section{Introduction}

The interstellar medium (ISM) is a dynamic environment in which gas cycles from a diffuse ionized state to dense star forming molecular clouds and back. Stars and supernovae provide energy to disrupt and ionize the gas and generate dynamical flows within and above the disk. Spiral density waves at the leading edge of the arm compress the ionized interarm gas and initiate one of the processes of cloud formation. The dynamics of the arm and the energy sources within the arm determine the distribution of different interstellar gas components within and above the disk. The study of the spiral density waves is thus important for understanding how clouds form and galaxies evolve. The spiral tangent regions are ideal laboratories to study the interaction of the interstellar gas and spiral density waves in the Milky Way as they provide a unique viewing geometry with large enough path lengths to detect the diffuse (atomic or ionized) spiral arm components. This unique viewing geometry of a spiral arm is illustrated in Fig. 1 for the Scutum tangency (adapted from Fig. 6 in Velusamy et al. 2015) in which the different gas layers, ionized, atomic, and molecular are shown along with their emission line profiles as a function of $V_{\mathrm{LSR}}$. These tangencies are located at $l \sim 31^{\circ}, 51^{\circ}, 284^{\circ}, 310^{\circ}, 328^{\circ}$, and $339^{\circ}$ (Vallée 2008), although there is some scatter, $\sim 4^{\circ}$, in the identification of the location of the tangency depending on which tracer is used.

The atomic and molecular components of the ISM in the spiral arms are well traced in emission by $\mathrm{H}$ I and $\mathrm{CO}$, respectively, but the photon dominated regions (PDR), CO-dark $\mathrm{H}_{2}$ and the ionized gas has been less well studied in emission owing to the difficulty of observing key tracers from the ground. The finestructure transition of ionized carbon, [C II], at $158 \mu \mathrm{m}$, traces almost all the warm $(T>35 \mathrm{~K})$ portions of the ionized ISM. However, [C II] alone cannot distinguish fully ionized hydrogen gas from weakly ionized gas (carbon ionized but hydrogen neutral). In contrast, nitrogen, with its $14.53 \mathrm{eV}$ ionization potential, has fine-structure lines, [N II], at $122 \mu \mathrm{m}$ and $205 \mu \mathrm{m}$ that arise only in highly ionized regions. Here we investigate the influence of the spiral arms on the distribution and dynamics of the highly ionized gas in and out of the plane by observing velocity resolved [N II] $205 \mu \mathrm{m}$ and [C II] $158 \mu \mathrm{m}$ emission in the Scutum spiral arm tangency $\left(l \sim 31^{\circ}\right)$, taken with the German Receiver for 


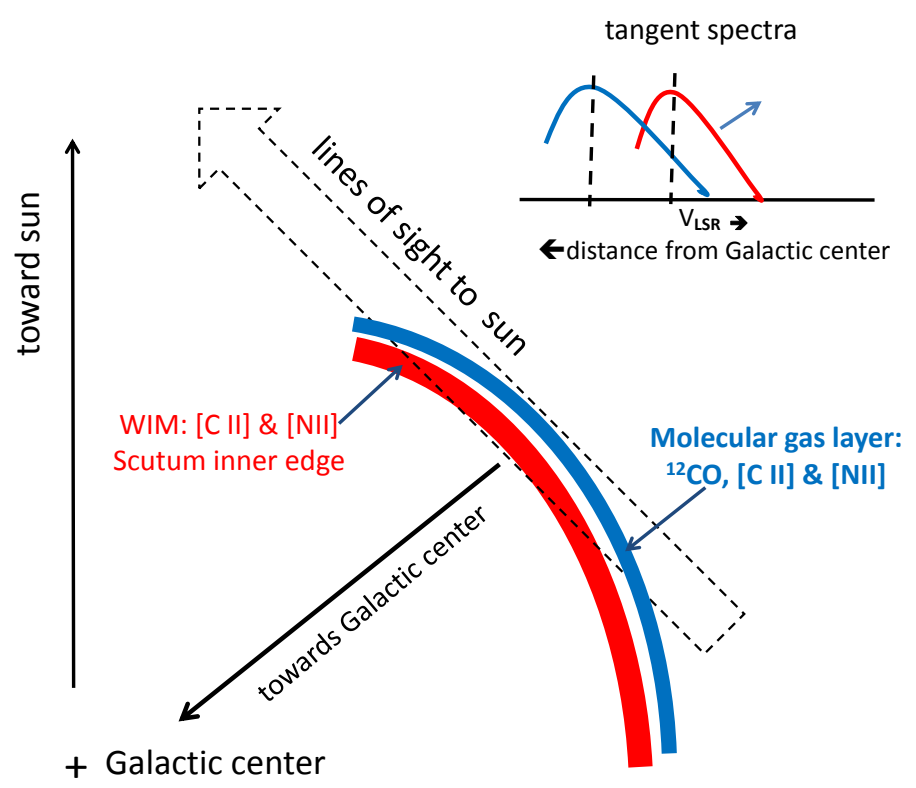

Fig. 1. Schematic view of the Scutum spiral arm tangency showing the structure of the gas layers (adapted from Fig. 6 in Velusamy et al. 2015). The emission of key gas tracers along the tangency, [C II], [N II], and CO help distinguish the WIM and molecular gas layers indicated as red and blue colors, respectively. Note the long path length along the tangency which improves the sensitivity to the weak [C II] and [N II] emission from the WIM. A schematic of the velocity $\left(V_{\mathrm{LSR}}\right)$ profile (shown in the inset) of the corresponding spectral line intensities for each layer, demonstrates why it is possible to separate the emission from the WIM from the neutral gas components. Note that this cartoon is intended to be a schematic and is not to scale.

Astronomy at Terahertz Frequencies (GREAT ${ }^{1}$; Heyminck et al. 2012) and the upGREAT ${ }^{1}$ array (Risacher et al. 2016), respectively, onboard the NASA/DLR Stratospheric Observatory for Infrared Astronomy (SOFIA; Young et al. 2012).

The large-scale structure of spiral arms in the Milky Way is a subject of great interest for understanding the dynamics of the Galaxy and for interpreting its properties. Modeling the Galactic spiral structure is based in part on data at the spiral arm tangents in different gas and stellar tracers. However, each of these tracers can occupy a separate lane across the arm reflecting the evolution of gas from low- to high-density clouds as they are swept into the arm's gravitational potential. Using Herschel HIFI [C II] maps along with H I and CO maps, Velusamy et al. (2012, 2015) showed that the gas in the Scutum, Crux, Norma, and Perseus arms was arranged in layers and revealed an evolutionary transition from lowest to highest density states. In particular the geometry of the tangencies made possible the detection of the low density WIM in [C II] and revealed a higher density WIM at the inner edge of the arm than in the interarm gas. They suggested this increase was a result of the compression of the WIM at the leading edge. Here we use both [C II] and [N II] observations of the Scutum arm tangency at $l \sim 31^{\circ}$ to study the interaction of the spiral arm potential with the ionized interarm gas.

This paper is organized as follows. In Sect. 2 we present the observations and data reduction, while in Sect. 3 we describe the distribution of [C II] and [N II] in the Scutum tangency. In Sect. 4 we derive the properties of the ionized gas, including density

1 GREAT and upGREAT are a development by the MPI für Radioastronomie and the KOSMA/Universität zu Köln, in cooperation with the MPI für Sonnensystemforschung and the DLR Institut für Planetenforschung.

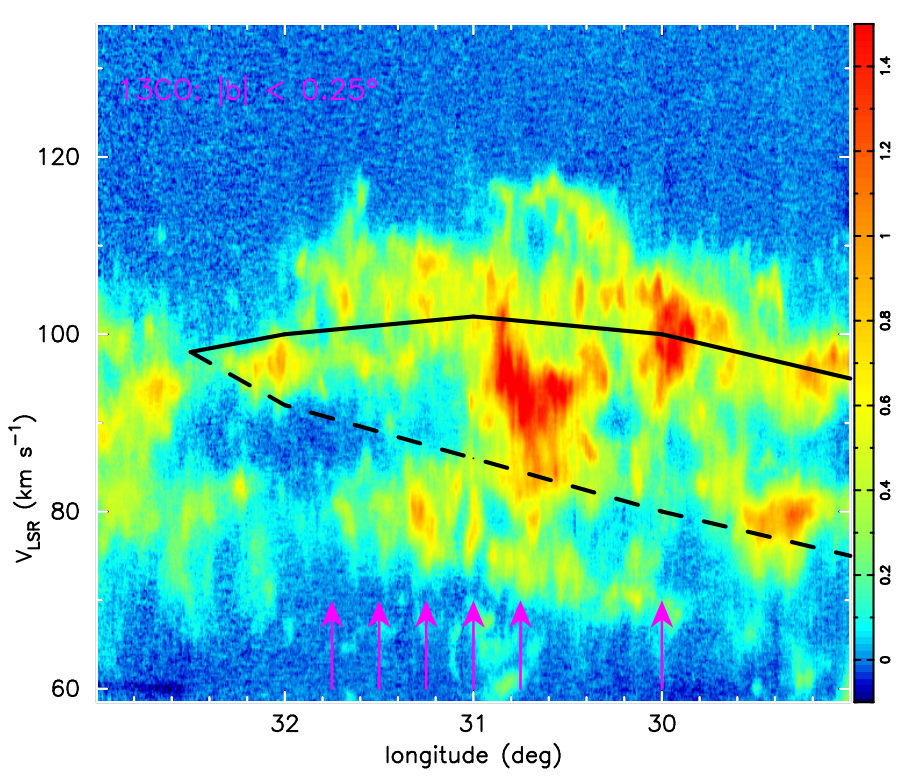

Fig. 2. A ${ }^{13} \mathrm{CO}$ longitude-velocity plot of the Scutum arm in the region $l \sim 29^{\circ}$ to $33^{\circ}$ at $b=0^{\circ}$, where we mark the inner (black solid line) and outer (dashed black line) tangencies taken from Reid et al. (2016) using their fit to the near-far distances. The arrows at the bottom indicate Galactic longitudes of the lines of sight observed in [C II] and [N II] with upGREAT and GREAT, respectively.

and scale height, using [C II] and [N II], and discuss the possible mechanisms responsible for the distribution of [N II]. Section 5 summarizes the results.

\section{Observations}

The Scutum arm is located about $4 \mathrm{kpc}$ from the Galactic Center and wraps more than half way around the Galaxy. In Fig. 2 we show a ${ }^{13} \mathrm{CO}$ longitude-velocity map of the tangency near $l \sim 31^{\circ}$ and have marked the inner and outer tangencies derived from Reid et al. (2016) with black lines. The ${ }^{13} \mathrm{CO}$ traces the molecular clouds in this region. The ${ }^{13} \mathrm{CO}\left(l-V_{\mathrm{LSR}}\right)$ map at Galactic latitude, $b=0^{\circ}$ is derived from the Galactic Ring Survey (GRS) data (Jackson et al. 2006).

To compare the spatial and velocity structure of the spiral arm gas components in both the molecular (neutral) and the ionized gases in the Scutum arm we made small-scale cross maps of $[\mathrm{C}$ II] and $[\mathrm{N}$ II] in longitude along and in latitude above a spiral arm tangency with the GREAT single pixel receiver for [N II] and the upGREAT 7-pixel array for [C II]. The [N II] and $\mathrm{C}$ [II] emission across the arm allows us to examine the impact of the spiral arm potential on the ionized gas components. To examine the spatial and velocity structures of these gas components across the tangency and perpendicular to the Galactic plane we observed [N II] and [C II] along 18 lines of sight (LOS) across the Scutum tangency from 30.0 to 31.75 covering $b$ from -0.25 to 1.7 . These 18 LOS are indicated in Fig. 3 by crosses superimposed on a $21 \mathrm{~cm}$ continuum map (Stil et al. 2006) of the Scutum tangency region. We have also indicated the primary, secondary, and tertiary reference sky positions used in the observations (see discussion below).

We observed the Scutum tangency in the ionized carbon $\left(\mathrm{C}^{+}\right){ }^{2} \mathrm{P}_{3 / 2}-{ }^{2} \mathrm{P}_{1 / 2}$ fine structure line, [C III], at $1900.5369 \mathrm{GHz}$ $(\lambda \sim 157.741 \mu \mathrm{m})$, using upGREAT (Risacher et al. 2016) and the ionized nitrogen $\left(\mathrm{N}^{+}\right){ }^{3} \mathrm{P}_{1}-{ }^{3} \mathrm{P}_{0}$ fine structure line, [N II], at $1461.1338 \mathrm{GHz}(\lambda \sim 205.178 \mu \mathrm{m})$ using GREAT 


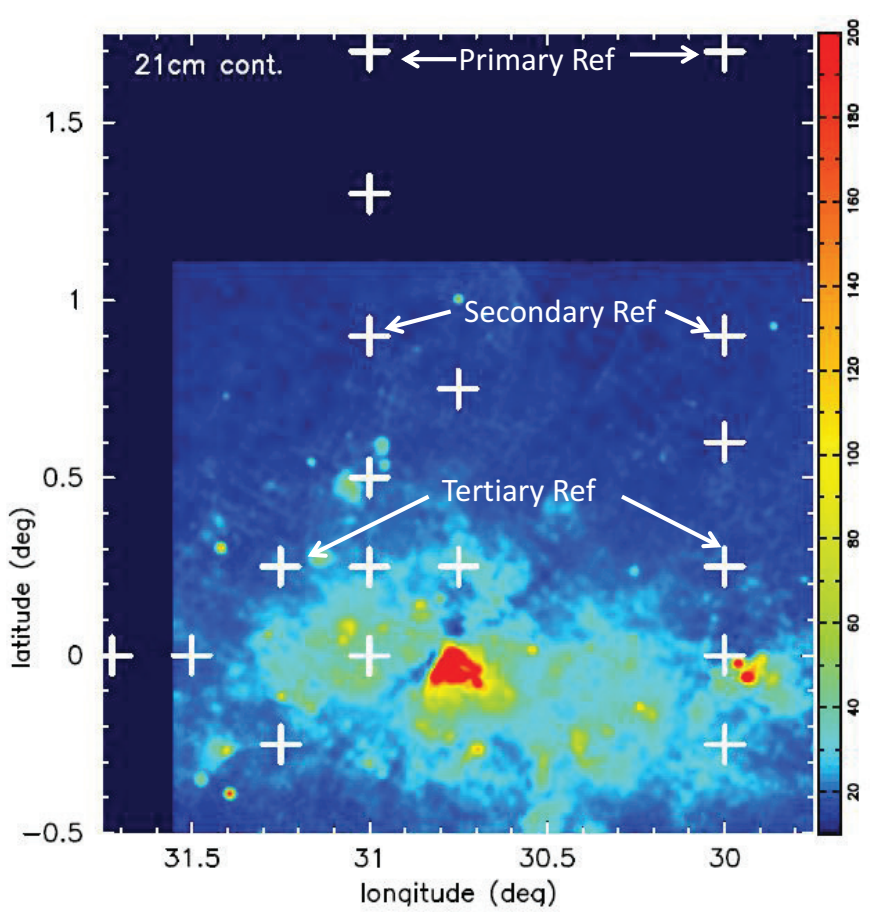

Fig. 3. The 18 lines of sight observed in [C II] and [N II] are indicated by white crosses superimposed on a $21 \mathrm{~cm}$ continuum map of the Scutum tangency region (Stil et al. 2006). The primary reference off positions are labeled as are the secondary and tertiary reference positions.

(Heyminck et al. 2012) onboard SOFIA (Young et al. 2012). The upper state of $\mathrm{C}^{+},{ }^{2} \mathrm{P}_{3 / 2}$, lies at an energy, $E / k$, about $91.2 \mathrm{~K}$ above the ground state, and the upper states of $\mathrm{N}^{+},{ }^{3} \mathrm{P}_{1}$ and ${ }^{3} \mathrm{P}_{2}$, lie at $70.1 \mathrm{~K}$ and $188.2 \mathrm{~K}$, respectively, above the ground state. Our program (proposal ID 04_0033; PI Langer) was part of the Guest Observer Cycle 4 campaign. The observations were made between May 12 and June 10, 2016 on six flights spread over SOFIA flights \#296 to \#308. Both [C II] and [N II] spectra were observed simultaneously in GREAT configuration: $\mathrm{LFA}+\mathrm{H}+\mathrm{V} / \mathrm{L} 1$ with $[\mathrm{CII}]$ in LFA:LSB and [N II] in L1:USB. The typical observing time on each target was in the range of 15 to $30 \mathrm{~min}$.

[C II] and [N II] are widespread throughout the Galaxy so finding a clean off position for proper calibration is a challenge in observing the Scutum spiral arm. The telescope on SOFIA cannot slew more than 0.4 to 0.8 from the ON to the OFF position, and it was thus necessary to observe [C II] and [N II] in a series of steps in $b$ starting at a latitude where the emission is weak enough to provide a clean OFF position. In practice even the largest $b$ value was not always absolutely clean of emission, but it was generally weak enough to use as a reference position. The observing scenario, therefore, used a set of primary, secondary and tertiary reference positions located at $b=1.7$, 0.9 , and 0.25 , respectively as shown in Fig. 3 . The primary reference positions, the LOSs at $b=1.7$, were observed with respect to an OFF position at $b=2.1$ at the same longitude. These LOS at $(l, b)=(30.0,1.7)$ and $(31.0,1.7)$ were then used as primary reference positions for observations at lower values of $b$, two of which at $b=0.9$ then could be used as secondary reference positions (see Fig. 3) for LOS at yet lower values of $b$. This procedure was repeated one more time and a set of tertiary reference positions were established at $b=0.75$ (see Fig. 3). In this scheme each target LOS used the nearest (up to

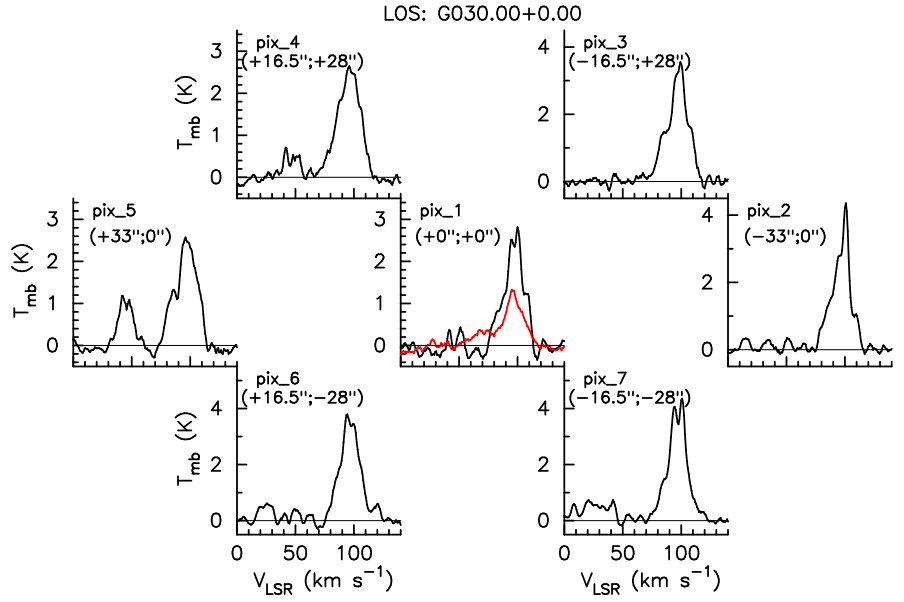

Fig. 4. The seven [C II] spectra observed by upGREAT (black) and the central [N II] spectrum (red) observed by GREAT towards $(l, b)=\left(30^{\circ} 0\right.$, 0.0 ). While the overall shapes of the [C II] lines across the Scutum spiral $\operatorname{arm}\left(V_{\mathrm{lsr}}=60\right.$ to $\left.130 \mathrm{~km} \mathrm{~s}^{-1}\right)$ within each pixel are similar, the small differences among them demonstrate that there is structure due to individual cloud components. The [N II] emission in the central pixel is detected across the entire velocity range of $[\mathrm{C} \mathrm{II}]$ in the Scutum arm.

within 0.8 ) primary/secondary/tertiary reference position. The calibrated emission spectra at the reference positions were then added incrementally in a hierarchical manner to the respective target spectra.

The dual-polarization 7-pixel upGREAT array has a FWHM beam size of $14^{\prime \prime}$ at $1.9 \mathrm{THz}$. The array is arranged in a hexagonal pattern with a central beam. The beam spacing is approximately two beam widths and the array has a footprint about $67 "$ across. At the distance to the Scutum arm tangency, $\sim 7 \mathrm{kpc}$ (see Sect. 4), each pixel corresponds to $\sim 0.5 \mathrm{pc}$ and the array stretches across $\sim 2.3 \mathrm{pc}$. The [N II] lines were observed with the GREAT receiver which has only one dual-polarization pixel with a FWHM beam size of $19^{\prime \prime}$ at $1.4 \mathrm{THz}$. The [N II] single pixel is centered on the central [C II] (pix_1), as shown in Fig. 4 for $(30.0,0 \circ 0)$. It can be seen that to first order the overall shape of the [C II] emission is similar across the array, however there are small scale variations reflecting the individual gas components. This variation on $20^{\prime \prime}$ scale is not surprising considering that the 7 -pixel footprint of upGREAT covers $\sim 2.3$ pc across, and probably includes a number of different cloud components. Only for the central pixel (pix_1) do both [C II] and [N II] have identical pointings. Therefore for all analysis comparing their intensities we use only the [C II] central pixel. For all other analysis of large scale or global characteristics of [C II] emission, for example in comparison to molecular gas traced by ${ }^{13} \mathrm{CO}$, we use the $[\mathrm{C}$ II] spectra averaged over all 7 pixels because this average roughly matches the beam sizes of the $\mathrm{CO}$ auxiliary data. The intensities have been converted to main beam temperature, $T_{\mathrm{mb}}(\mathrm{K})$, using beam efficiencies, $\eta_{\mathrm{mb}}([\mathrm{C}$ II $])=0.65$ and $\eta_{\mathrm{mb}}([\mathrm{N} \mathrm{II}])=0.66$ with a forward efficiency, $\eta_{\text {for }}=0.97$ (Röllig et al. 2016). The rms noise for $T_{\mathrm{mb}}(\mathrm{K})$ in the calibrated spectra shown in Fig. 5 are in the range of 0.05 to $0.17 \mathrm{~K}$ for [N II], 0.04 to $0.25 \mathrm{~K}$ for [C II] averaged over 7 pixels, and 0.08 to $0.30 \mathrm{~K}$ in pixel \#1. To avoid the propagation of noise from the off position, we add to the target spectrum only off source spectral intensities that are above 1.5 times the rms noise. This approach is particularly important to minimize adding noise to the spectra since a majority of the target LOS spectra use multiple off source spectra for reference. The large variation in the noise levels in these spectra are due to the differences in the observing duration used for each LOS. 

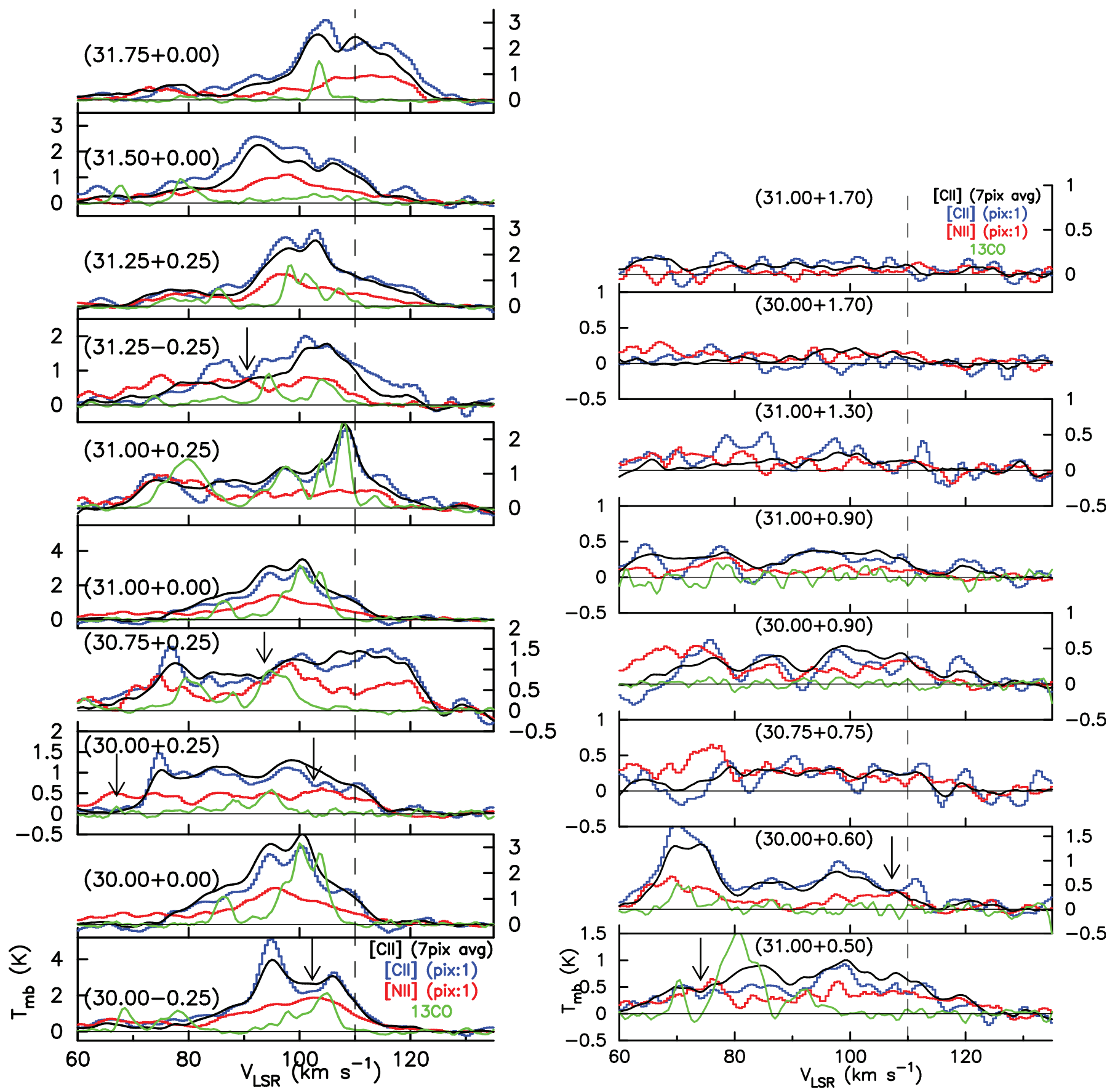

Fig. 5. Left: emission spectra of [C II] (blue and black), [N II] (red), and ${ }^{13} \mathrm{CO}$ (green) for the ten lines of sight that are within $b= \pm 0: 25$ are plotted as $T_{\mathrm{mb}}$ versus velocity. The central [C II] pixel is plotted in blue and the average of the seven [C II] pixels is plotted in black. The single and average [C II] emission profiles are similar but not exactly identical owing to variations in emission across the footprint of the upGREAT array. The black vertical dashed line marks the tangent velocity of the Scutum spiral arm. Right: same as in the left panel for the eight lines of sight with $b>0: 25$. The downward black arrows mark a few of the clearly evident [C II] absorption features.

In addition to the SOFIA [C $\mathrm{II}]$ and $[\mathrm{N} \mathrm{II}]$ observations we use auxiliary $\mathrm{HI}$ and ${ }^{13} \mathrm{CO}(1-0)$ data from public archives, [N II] from Herschel PACS and HIFI at (30:0, 0:0) and (31:2766, 0:0) (Goldsmith et al. 2015; Langer et al. 2016), and [C II] with HIFI (Langer et al. 2016). The H I $21 \mathrm{~cm}$ data are taken from the VLA Galactic plane survey (VGPS; Stil et al. 2006). The ${ }^{13} \mathrm{CO}(1-$ 0) data from the Galactic Ring Survey (GRS; Jackson et al. 2006). We extracted the spectra for the LOSs observed by SOFIA from these survey data, which are available as Galactic longitude-latitude- $V_{\text {LSR }}$ spectral line data cubes. Both VGPS and GRS surveys have comparable angular resolution of $1^{\prime}$ and $40^{\prime \prime}$, respectively. The spectral resolution in the HI VGPS is $1.56 \mathrm{~km} \mathrm{~s}^{-1}$ with rms noise of $2 \mathrm{~K}$ and in the ${ }^{13} \mathrm{CO}$ GRS the spectral resolution is $0.21 \mathrm{~km} \mathrm{~s}^{-1}$ with rms of $2 \mathrm{~K}$.

\section{Results}

In this section we describe the characteristics of the spectral features and the morphology of the Scutum tangency traced by [C II] and [N II]. We use the $V_{\mathrm{LSR}}$ velocity structure in the line profiles to infer that the spiral arm gas components are roughly distributed in different layers of the arm ranging from 
low density WIM to the dense molecular gas as in the illustration of the distribution of ISM spiral arm gas components shown in Fig. 1.

These layers display the gas evolutionary sequence whereby the WIM falls into the gravitational potential, recombines, and is successively converted to higher density components, diffuse atomic, diffuse molecular, and dense molecular clouds, by either compression, cloud collisions, or convergent flows. The tangencies are unique regions where the structural layers and dynamics of the arm can be more readily separated. Furthermore, we use the velocity distribution to separate the WIM from the neutral layers. Thus by observing this region spectroscopically we can sample the different gas layers of the arm, and separate the WIM's [N II] and [C II] emission from the other ionized components.

\section{1. [C II] and [NII] spectra}

In Fig. 5 (left panel) we plot the main beam temperature, $T_{\mathrm{mb}}$, for [C II], [N II], and ${ }^{13} \mathrm{CO}(1-0)$ versus $V_{\mathrm{LSR}}$ for ten lines of sight across the Scutum arm $\left(V_{\mathrm{LSR}} \sim 60\right.$ to $\left.120 \mathrm{~km} \mathrm{~s}^{-1}\right)$, from longitude $l=30.0$ to 31.75 and for $b$ within \pm 0.25 . In Fig. 5 (right panel) we plot these tracers for the eight lines of sight with $b>0.25$. For each LOS the red and blue lines show [N II] and the central pixel of [C II], respectively, while the black line shows the average of the 7 [C II] pixels. The green lines show the ${ }^{13} \mathrm{CO}(1-0)$ profile, which is used as a proxy for the velocity structure of the denser molecular spiral arm gas component. The [C II] traces both neutral and ionized gas components while [N II] traces only the ionized gas. The vertical dashed line indicates the tangent velocity as derived from assuming a Galactic rotation model (e.g. Roman-Duval et al. 2009).

In general the [C II] central pixel and 7-pixel average are similar but there are small differences, as was also shown in the comparison of individual [C II] pixels in Fig. 4. The [C II] central pixel (blue) and [N II] (red) profiles represent emissions observed with comparable beams of $14^{\prime \prime}$ and $18^{\prime \prime}$ sizes, respectively, which allows a direct comparison of their intensities. On the other hand, the [C II] 7 pixel average profiles (black) correspond to emission smoothed to a larger angular beam, $\sim 60^{\prime \prime}$, comparable to the beam size of the ${ }^{13} \mathrm{CO}\left(\sim 45^{\prime \prime}\right)$ and H I $\left(\sim 60^{\prime \prime}\right)$ data. To display the velocity structure within the Scutum arm more clearly in Fig. 5 we plot only the velocity range of the Scutum arm, $V_{\mathrm{LSR}}=60-130 \mathrm{~km} \mathrm{~s}^{-1}$.

Comparison of the intensities of the [C II $], I([\mathrm{C}$ II $])$, central pixel (blue) to $[\mathrm{N} \mathrm{II}], I([\mathrm{NII}])$, (red) as a function of $V_{\mathrm{LSR}}$ show regions where $I([\mathrm{C} I I])$ to $I([\mathrm{~N} \mathrm{II}])$, is less than 1.5 , including some velocities where [N II] is stronger than [C II]. Analysis of the predicted contribution of [C $\mathrm{II}]$ from [N II] regions (see Sect. 4.2) shows that this ratio can not be less than 1.7 and more typically should be greater than 2 . The low ratio seen at some velocities in Fig. 5 is a result of foreground and/or self-absorption. We have indicated a few of these absorption regions with downward arrows. Evidence for [C II] absorption, sometimes strong absorption has been noted by Langer et al. (2016) towards other LOS observed in [N II] and [C II] with HIFI.

\subsection{Longitudinal and latitudinal distribution}

The [N II] and [C II] emission for the spectra within $|b|<0.25$ covering $l=30.0$ to 31.75 in the Scutum tangency, as shown in Fig. 5, extend over the same velocity range. In contrast, ${ }^{13} \mathrm{CO}$ is present only over portions of the velocity range of the Scutum tangency. The total emission is a blend of several cloud components as can be seen from the complexity in the ${ }^{13} \mathrm{CO} l-V_{\mathrm{LSR}}$ map in Fig. 2. While the [N II] distribution approximately follows that of [C II], the ratio of $T_{\mathrm{mb}}$ of [C II] to [N II] varies with $V_{\mathrm{LSR}}$. In some lines of sight the [N II] emission is nearly as strong as [C II]. This association is seen in other studies of [N II] and [C II] and, as noted above, is due in part to [C II] absorption by low excitation foreground material (Langer et al. 2015).

The latitudinal distribution of the emission shown in Fig. 5 (right panel) reveals that ${ }^{13} \mathrm{CO}$ is virtually absent for $b>0.6$ but that [C II] and [N II] extend at least up to $b=1.3$ and perhaps to 1:7. Closer to the plane, the emission from [N II] and [C II] extend across the entire velocity range of the Scutum tangency, while ${ }^{13} \mathrm{CO}$ is restricted to a few velocity components. Taken together, the longitudinal and latitudinal distributions indicate that highly ionized gas, as traced by [N II] and [C II], and weakly ionized gas, as traced by a portion of [C II] are present throughout all layers of the Scutum arm tangency.

\subsection{Velocity structure of spiral arm gas components}

To characterize the overall distribution of gas across the Scutum arm we averaged the spectra from all longitudes $(l=30.0$ to 31.75 ) whose lines of sight fall within $b= \pm 0.25$. This averaging improves the signal-to-noise of the weak WIM emission and avoids biasing the results by observations towards any compact regions. In Fig. 6 we plot the resulting $T_{\mathrm{mb}}$ from the atomic, molecular, and ionized gas tracers versus $V_{\mathrm{LSR}}$. The top panel in Fig. 6 compares $T_{\mathrm{mb}}$ versus $V_{\mathrm{LSR}}$ over the full velocity range of the Scutum tangency (note only the central upGREAT pixel is used so that $[\mathrm{C}$ II $]$ and $[\mathrm{N}$ II $]$ correspond to the same LOS). The shaded rectangle $\left(V_{\mathrm{LSR}}=98\right.$ to $\left.125 \mathrm{~km} \mathrm{~s}^{-1}\right)$ designates the emission from the inner Scutum arm where the different gas layers are more easily separated. It is clear that the ${ }^{13} \mathrm{CO}$ emission does not extend beyond $\sim 110 \mathrm{~km} \mathrm{~s}^{-1}$ while [C II] and [N II] extend up to at least $125 \mathrm{~km} \mathrm{~s}^{-1}$. Thus the [C II] and [N II] extend outside the edge of the molecular gas tangency. The [N II] emission beyond $110 \mathrm{~km} \mathrm{~s}^{-1}$ likely arises primarily from the highly ionized gas at the inner edge of the Scutum arm.

To calculate the distribution and properties of the highly ionized gas we need to isolate the velocities over which [N II] and [C II] characterize this gas alone versus the emission that comes from PDRs associated with the ${ }^{13} \mathrm{CO}$ molecular gas. It has been shown by Velusamy et al. (2015) that the emissions in the gas components trace different gas layers which are well separated in $V_{\mathrm{LSR}}$ across the spiral arm tangency. To separate these components we made a multi-Gaussian fit to the composite [N II], the $[\mathrm{C} \mathrm{II}]$ central pixel, and ${ }^{13} \mathrm{CO}$ spectra in Fig. 6 across the velocity range 60 to $130 \mathrm{~km} \mathrm{~s}^{-1}$. The high end of this velocity range represents the WIM at the inner edge of the arm and it is highlighted by the shaded box in the upper panel of Fig. 6. To separate out any [C II] or [N II] contribution in this box that is associated with the molecular gas traced by ${ }^{13} \mathrm{CO}$, we use the Gaussian fits to ${ }^{13} \mathrm{CO}$ to isolate the molecular components, as follows. We fit the [C II] and [N II] emission by adjusting the peak intensity of a set of Gaussian profiles associated with the gas at velocities $<110 \mathrm{~km} \mathrm{~s}^{-1}$, keeping their $V_{\mathrm{LSR}}$ at the peak and FWHM the same as that for ${ }^{13} \mathrm{CO}$. In the lower panel of Fig. 6 we show the two highest velocity ${ }^{13} \mathrm{CO}$ Gaussian components within the box. We then subtracted out the respective Gaussian components for $[\mathrm{C} \mathrm{II}]$ and $[\mathrm{N}$ II $]$ and plot the remaining excess in the right hand sub-panel, which represents just the [C II] and [N II] contributions from the highly ionized gas at the innermost edge of the Scutum arm. 


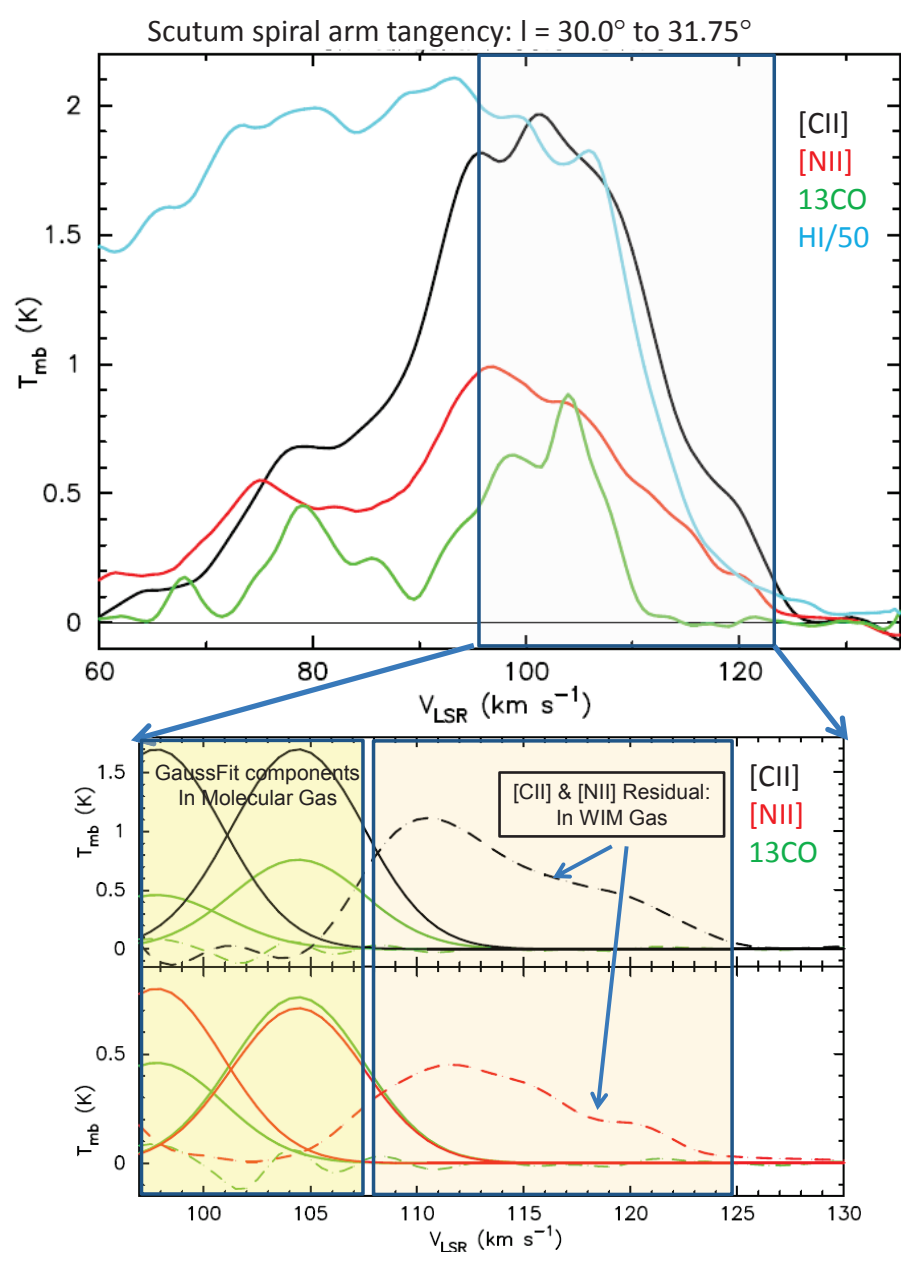

Fig. 6. Top: spectra for the atomic, molecular, and ionic gas tracers, H I (blue), ${ }^{13} \mathrm{CO}$ (green), [C II] (black), and [N II] (red), averaged over all LOS within $b= \pm 0.25$ plotted as main beam temperature, $T_{\mathrm{mb}}$, versus $V_{\text {LSR }}$. The ionized gas tracers are seen to extend to much higher velocities, corresponding to the innermost leading edge of the Scutum arm, than the molecular gas as traced by ${ }^{13} \mathrm{CO}$. Bottom: expanded view of the ionized gas emission after subtracting out the [C $\mathrm{II}]$ and [N II] emission arising from the molecular clouds traced by ${ }^{13} \mathrm{CO}$. The solid green curves represent two of the Gaussian components fit to the ${ }^{13} \mathrm{CO}$ emission. The dashed green curve is the residual ${ }^{13} \mathrm{CO}$ after subtraction. Gaussian profiles were used to fit the $[\mathrm{C} \mathrm{II}]$ and $[\mathrm{N} \mathrm{II}]$ emission associated with the gas at velocities $<110 \mathrm{~km} \mathrm{~s}^{-1}$, while keeping their $V_{\mathrm{LSR}}$ at the peak and FWHM the same as the ${ }^{13} \mathrm{CO}$, and only adjusting their peak intensities. Thus what remains for $V_{\mathrm{LSR}} \geq 110 \mathrm{~km} \mathrm{~s}^{-1}$ is the [C II] and [N II] excess arising solely from the highly ionized gas with no associated $\mathrm{CO}$.

The strong correlation between the [C II] and [N II] emission in the right-subpanel is indicative of [C II] emission arising from ionized gas with negligible contribution from neutral H I gas (see Sect. 4.2). The two shaded regions in the lower panel represent schematically the molecular gas (left) and WIM gas (right). The fact that the ${ }^{13} \mathrm{CO}$ residual (dashed green line) is negligible shows that the emission in all gas components within the molecular gas layers of the Scutum spiral arm are well accounted for by the two Gaussian profiles (green lines) with linewidths $(F W H M) \sim 7-8 \mathrm{~km} \mathrm{~s}^{-1}$ and centered at $V_{\mathrm{LSR}} \sim 98$ and $105 \mathrm{~km} \mathrm{~s}^{-1}$, respectively. Therefore, we conclude that the $[\mathrm{C} \mathrm{II}]$ and $[\mathrm{N}$ II] residuals (black and red profiles, respectively) in the lower panel clearly delineate WIM gas that is well isolated from the molecular spiral arm. Note that the two Gaussian fits to the molecular spiral arm are also consistent with ${ }^{13} \mathrm{CO}$ in the $\left(l-V_{\mathrm{LSR}}\right)$ map shown in Fig. 2.

\section{Discussion}

Our SOFIA observations provide a rich data set of velocity resolved [C II] and [N II] emissions in the Scutum tangency. Our results show widespread clear detections of [C II] and [N II] along all LOSs, especially, at velocities $V_{\mathrm{LSR}}=60$ to $125 \mathrm{~km} \mathrm{~s}^{-1}$ and at $|b|<0.25$. Although weaker at higher latitudes there is detectable emission at least up to $b=1.3$. The unique combination of [C II] and [N II] provides a powerful tool to analyze the ionized gas component in spiral arms and the ability to isolate [C II] emission in the neutral and ionized gases.

In general the WIM is a highly ionized, low density, $n(\mathrm{e}) \sim$ 0.02 to $0.1 \mathrm{~cm}^{-3}$, high temperature, $T_{\mathrm{k}}=6000$ to $10000 \mathrm{~K}$ gas that fills a 2-3 kpc layer around the Galactic midplane (cf. Ferrière 2001; Cox 2005; Haffner et al. 2009). It is estimated to contain about $90 \%$ of the ionized gas in the Galaxy and has a filling factor of order 0.2 to 0.4 , which may depend on height above the plane. At the midplane the filling factor may be as small as $10 \%$. It has been studied with pulsar dispersion measurements (Cordes \& Lazio 2003), H $\alpha$ emission (Haffner et al. 2009, 2016), and nitrogen emission lines in the visible (Reynolds et al. 2001). More recently the WIM has been detected in absorption with [N II] (Persson et al. 2014). The [C II] and [N II] emission lines can also be used to map the WIM, however, the low densities of the WIM make it difficult to detect their emission throughout most of the Galaxy. However, the spiral arm tangencies offer a unique geometry that enables the detection of the WIM in [N II] and $[\mathrm{C} \mathrm{II}]$ because they correspond to regions with a very long path length in a relatively narrow velocity dispersion. The WIM has been detected in [C II] emission by Velusamy et al. (2012, 2015) along the tangencies of the Perseus, Norma, Scutum, and Crux arms, and they derive higher densities than the interarm WIM. Clearly the WIM in spiral arms is different from that throughout the Galactic disk. In this section we investigate the extended properties of the warm ionized medium as traced by [C II] and [N II] in the tangency region of the Scutum arm.

For the analysis and interpretation of the [C II] and [N II] emission we adopt a distance of $\sim 7 \mathrm{kpc}$ for the Scutum tangency estimated using the observed $V_{\text {LSR }}$ and the Galactic rotation model. In Fig. 7 we show the distance- $V_{\text {LSR }}$ relationship for the Galactic longitude of the Scutum tangency $(l=31 \% 0)$ derived using the rotation curve given by Reid et al. (2014) in their Fig. 4 and the Galactic rotation parameters for their Model A5. The rotation velocities in this model for Galactocentric distance, $R_{\mathrm{G}}>4 \mathrm{kpc}$, is quite valid for the location of the Scutum tangency. The WIM component observed at $V_{\mathrm{LSR}}>110 \mathrm{~km} \mathrm{~s}^{-1}$ is located at the distance of the tangent points, $\sim 7 \mathrm{kpc}$ and extends about $\pm 1 \mathrm{kpc}$. We note that the distance $-V_{\mathrm{LSR}}$ relationship plotted in Fig. 7 yields a distance of $5.5 \mathrm{kpc}$ for the star forming region $\mathrm{W} 43$ (for $V_{\mathrm{LSR}}=95-110 \mathrm{~km} \mathrm{~s}^{-1}$ ) which is consistent with a distance of $5.5 \mathrm{kpc}$, obtained from maser parallaxes (Zhang et al. 2014). Thus the Galactic bar-spiral arm interaction region containing W43 appears to be just outside the outer part of the Scutum tangency (see Fig. 9).

\subsection{Electron density of the Scutum WIM}

Nitrogen ions have two fine structure transitions, ${ }^{3} \mathrm{P}_{1} \rightarrow{ }^{3} \mathrm{P}_{0}$ at $205 \mu \mathrm{m}$ and ${ }^{3} \mathrm{P}_{2} \rightarrow{ }^{3} \mathrm{P}_{1}$ at $122 \mu \mathrm{m}$, and these can be used with an excitation model to calculate the electron density and column density $N\left(\mathrm{~N}^{+}\right)$. The $122 \mu \mathrm{m}$ line needs to be observed from space 
W. D. Langer et al.: Ionized gas in the Scutum spiral arm as traced in [N II] and [C II]

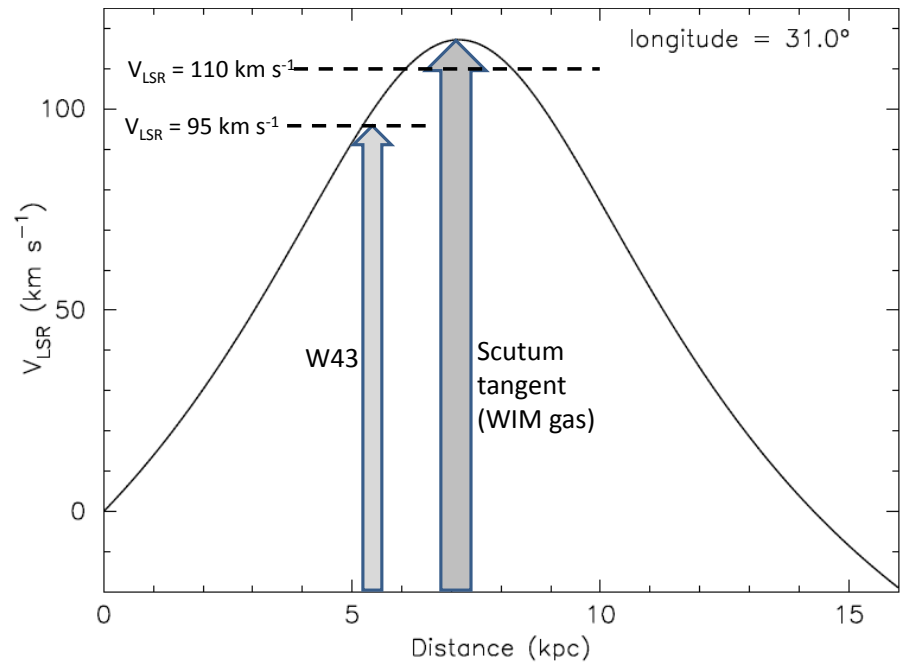

Fig. 7. The distance- $V_{\mathrm{LSR}}$ relationship for the Scutum arm tangency at $l=31.0$ derived using the rotation curve of Reid et al. (2014). It shows that the WIM component of the Scutum tangency is from 6$8 \mathrm{kpc}$, centered at $\sim 7 \mathrm{kpc}$ distance, while the prominent star forming and $\mathrm{H}$ II region, W43, is at about $5.5 \mathrm{kpc}$.

or a stratospheric balloon due to atmospheric opacity. As we only have one [N II] transition towards the Scutum tangency observed from SOFIA we cannot determine density $n(\mathrm{e})$ and column density $N\left(\mathrm{~N}^{+}\right)$independently, however, we can get an idea of the range of $n(\mathrm{e})$ using an approach outlined in Langer et al. (2015) that depends on having an estimate of the size of the emission region. For optically thin emission from a uniform region the intensity of the ${ }^{3} \mathrm{P}_{1} \rightarrow{ }^{3} \mathrm{P}_{0}$ transition can be written (Langer et al. 2015),

$$
I_{\mathrm{ion}}([\mathrm{N} \mathrm{II}])=0.156 x_{-4}\left(\mathrm{~N}^{+}\right) L_{\mathrm{pc}} n(\mathrm{e}) f_{1}\left(n(\mathrm{e}), T_{\mathrm{k}}\right)\left(\mathrm{K} \mathrm{km} \mathrm{s}^{-1}\right)
$$

where $I_{\text {ion }}([\mathrm{N} \mathrm{II}])$ is the intensity in $\mathrm{K} \mathrm{km} \mathrm{s}^{-1}, x_{-4}\left(\mathrm{~N}^{+}\right)$is the fractional abundance of $\mathrm{N}^{+}$in units of $10^{-4}, L_{\mathrm{pc}}$ is the size of the emission region in $\mathrm{pc}, T_{\mathrm{k}}$ is the kinetic temperature, and $f_{1}(n(\mathrm{e})$, $T_{\mathrm{k}}$ ) is the fractional population of the ${ }^{3} \mathrm{P}_{1}$ state, which is a function of $T_{\mathrm{k}}$ and $n(\mathrm{e})$. In the optically thin limit one can solve exactly for the fractional population of the levels as a function of $n$ (e) for a given kinetic temperature (Goldsmith et al. 2015). At the high kinetic temperatures associated with highly ionized gas, the kinetic energy of the electrons is orders of magnitude larger than the excitation energies required to populate the ${ }^{3} \mathrm{P}_{1}$ and ${ }^{2} \mathrm{P}_{3 / 2}$ states and the solutions are only weakly dependent on $T_{\mathrm{k}}$ through the collisional rate coefficients. For example for the collisional de-excitation rate coefficients calculated by Tayal (2011) the temperature dependence varies from $\sim T_{\mathrm{k}}^{-0.3}$ to $T_{\mathrm{k}}^{-0.5}$ depending on the transition.

Equation (1) can be solved iteratively for $n(\mathrm{e})$ as a function of $I_{\text {ion }}([\mathrm{N}$ II $])$, using the balance equations for the population of the ${ }^{3} P_{2}$ and ${ }^{3} P_{1}$ (see Goldsmith et al. 2015), assuming a reasonable value of $T_{\mathrm{k}}$, and given the size of the emission region and the fractional abundance of $\mathrm{N}^{+}$. The critical density (where the collisional de-excitation rate equals the radiative rate) for the ${ }^{3} \mathrm{P}_{1}$ level, $n_{\mathrm{cr}}(\mathrm{e})$, is $\sim 175 \mathrm{~cm}^{-3}$, so that at low densities, $n(\mathrm{e}) \ll n_{\mathrm{cr}}(\mathrm{e})$, Eq. (1) can also be solved approximately, within $\pm 15 \%$, using,

$n(e)=a_{0}\left[\frac{I([\mathrm{~N} \mathrm{II}])}{x_{-4}\left(\mathrm{~N}^{+}\right) L_{\mathrm{pc}}}\right]^{\alpha}\left(\mathrm{cm}^{-3}\right)$, where, at a characteristic WIM temperature, $T_{\mathrm{k}}=8000 \mathrm{~K}, a_{0}=$ 18.0 and $\alpha=0.51$ for $n(\mathrm{e}) \leq 11 \mathrm{~cm}^{-3}$, and $a_{0}=22$ and $\alpha=0.72$, for $11<n(\mathrm{e}) \leq 100 \mathrm{~cm}^{-3}$.

We consider the average properties of two Galactic zones, one within the plane and the other above the plane. In the plane we average over all LOS within $b= \pm 0.25$, and above the plane we average all LOS within $b=0.60$ to 0.90 (we exclude the LOS at $b=1.3$ and 1.7 because they are too noisy). By using all the data within these two regions we improve the signal to noise and minimize the effects of any localized sources. We adopt a value for the fractional abundance $x\left(\mathrm{~N}^{+}\right)=1.4 \times 10^{-4}$ (see Sect. 6.3.2 Goldsmith et al. 2015), appropriate for the Galactic radius, $R_{\mathrm{G}}=4 \mathrm{kpc}$. From the geometry of the Scutum outer arm (see Fig. 9 and Reid et al. 2016) the range $l \sim 30^{\circ}$ to $32^{\circ}$ corresponds to a path length through the tangency $\sim 1 \pm 0.5 \mathrm{kpc}$, so we adopt $L=1 \mathrm{kpc}$ to estimate $n(\mathrm{e})$. (In the low density case (see Eq. (2)) $n(\mathrm{e})$ is $\propto L^{-0.5}$ so in choosing $L=1 \mathrm{kpc}$ the uncertainty in $n(\mathrm{e})$ is of order $\pm 25 \%$.) We solved for $n(\mathrm{e})$ as a function of $I_{\text {ion }}$ ([N II] ) over the velocity range 110 to $125 \mathrm{~km} \mathrm{~s}^{-1}$. We list the intensities and $\langle n(\mathrm{e})\rangle$ in Table 1. Typical average densities in the plane, are $\langle n(\mathrm{e})\rangle \sim 0.9 \mathrm{~cm}^{-3}$ and above the plane $\langle n(\mathrm{e})\rangle \sim 0.4 \mathrm{~cm}^{-3}$.

We can also calculate the density of the WIM from [C II], providing that we have managed to isolate its WIM contribution from other sources (PDRs, CO-dark $\mathrm{H}_{2}$, $\mathrm{H}$ I clouds), using an approach similar to that derived for [N II]. For $n(\mathrm{e})$ less than the critical density for exciting the ${ }^{2} \mathrm{P}_{3 / 2}$ level of $\mathrm{C}^{+}, n_{\mathrm{cr}}(\mathrm{e}) \sim$ $45 \mathrm{~cm}^{-3}$, the electron density is given by (see Velusamy et al. 2012),

$n(e)=2.92 T_{\mathrm{k}}^{0.18}\left[\frac{I([\mathrm{C} \mathrm{II}])}{x_{-4}\left(\mathrm{C}^{+}\right) \mathrm{L}_{\mathrm{pc}}}\right]^{0.5}\left(\mathrm{~cm}^{-3}\right)$.

To solve for $n(\mathrm{e})$ from [C II] we adopt $T_{\mathrm{k}}=8000 \mathrm{~K}$ (Eq. (3) is a very weak function of $T_{\mathrm{k}}$ ) and $x\left(\mathrm{C}^{+}\right)=2.9 \times 10^{-4}$ (see Eq. (2) in Pineda et al. 2013) appropriate to $R_{\mathrm{G}}=4 \mathrm{kpc}$. We only use the central [C II] pixel so that we are making a direct comparison of the $[\mathrm{N} \mathrm{II}]$ and $[\mathrm{C} \mathrm{II}]$ along the same LOS. We find typical values in the plane, $|b| \leq 0.25,\langle n(\mathrm{e})\rangle \sim 0.8 \mathrm{~cm}^{-3}$ and above the plane, $\sim 0.3 \mathrm{~cm}^{-3}$, similar to those derived from [N II] .

\section{2. [C II] from the Scutum WIM}

As shown in Langer et al. (2016) we can calculate how much [C II] emission comes from the highly ionized gas, $I_{\text {ion }}([\mathrm{C}$ II $]$ ), in the optically thin limit from the intensity of [N II], using the following relationship,

$I_{\text {ion }}([\mathrm{C} \mathrm{II}])=0.675 \frac{f_{3 / 2}\left(\mathrm{C}^{+}\right)}{f_{1}\left(\mathrm{~N}^{+}\right)} \frac{x\left(\mathrm{C}^{+}\right)}{x\left(\mathrm{~N}^{+}\right)} I_{\text {ion }}(\mathrm{N}$ II $)$,

where $f_{i}$ is the fractional population of the corresponding levels. In Eq. (4) $f_{3 / 2} / f_{1}$ is only weakly dependent on density for $n(\mathrm{e})=$ $10^{-3}$ to $10^{2} \mathrm{~cm}^{-3}$, and ranges from 1.22 to $1.73^{2}$, and we adopt $x\left(\mathrm{C}^{+}\right) / x\left(\mathrm{~N}^{+}\right)=2.1^{3}$.

2 At low densities, $n(\mathrm{e}) \leq 5 \mathrm{~cm}^{-3}, f_{3 / 2} / f_{1}$ ranges from 1.53 to 1.73 and to a good approximation we can set this ratio to a constant of 1.63 to within $\pm 6 \%$. The fractional abundance ratio, $x\left(\mathrm{C}^{+}\right) / x\left(\mathrm{~N}^{+}\right)=2.1$ yields a simple estimate for the [C II] emission arising from the highly ionized gas traced by $[\mathrm{N} \mathrm{II}], I_{\text {ion }}([\mathrm{C} \mathrm{II}])=2.3 I_{\text {ion }}([\mathrm{N} \mathrm{II}])$, when $n(\mathrm{e}) \leq 5 \mathrm{~cm}^{-3}$.

3 We adopt a solar fractional abundance of $x\left(\mathrm{~N}^{+}\right)=6.76 \times 10^{-5}$ (Asplund et al. 2009) with an abundance gradient -0.07 dex $\mathrm{kpc}^{-1}$ (Shaver et al. 1983), and for $\mathrm{C}^{+}, x\left(\mathrm{C}^{+}\right)=1.4 \times 10^{-4}$, with the same gradient as $x\left(\mathrm{~N}^{+}\right)$(Pineda et al. 2013), which yields a ratio, $x\left(\mathrm{C}^{+}\right) / x\left(\mathrm{~N}^{+}\right)=$ 2.1 . 

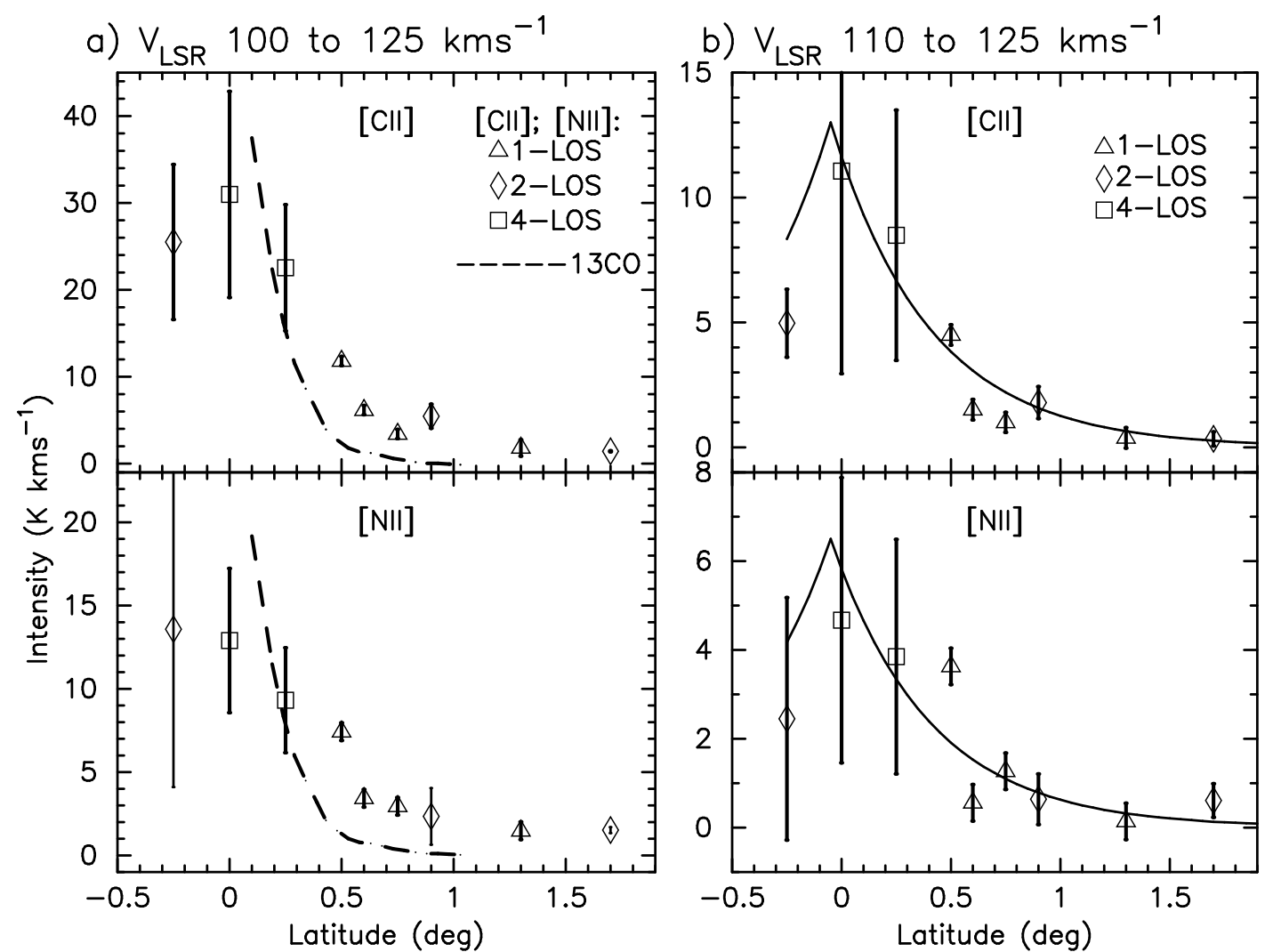

Fig. 8. Left panels: integrated intensities of [C II], [N II], and ${ }^{13} \mathrm{CO}$ (dashed line) over the velocity range 100 to $125 \mathrm{~km} \mathrm{~s}^{-1}$ versus latitude. The symbols indicate how many SOFIA LOS were averaged to calculate the emission intensity. The ${ }^{13} \mathrm{CO}$ intensity profile is computed using the GRS data cube and we show only high latitude values to demonstrate how sharply it drops to nearly zero by $b=0.5$. ${ }^{13} \mathrm{CO}$ has a much smaller scale height than [C II] and [N II]. Right panels: integrated intensities of [C II] and [N II] over the velocity range 110 to $125 \mathrm{~km} \mathrm{~s}^{-1}$ versus latitude. There is no measurable ${ }^{13} \mathrm{CO}$ in this velocity range, and the $[\mathrm{CII}]$ and $[\mathrm{N} \mathrm{II}]$ arise solely from the WIM. The exponential curves correspond to $\exp \left(-(b-\delta b) / b_{0}\right)$ with $\delta b=-0.05$ and $b_{0}=0.45$.

Table 1. Properties of the Scutum arm ionized gas.

\begin{tabular}{ccccccccccc}
\hline \hline $\begin{array}{c}b \text { range } \\
\text { averaged }\end{array}$ & $\begin{array}{c}V_{\mathrm{LSR}} \\
\mathrm{km} \mathrm{s}^{-1}\end{array}$ & $\begin{array}{c}I_{\text {ion }}([\mathrm{N} \mathrm{II}]) \\
\mathrm{K} \mathrm{km} \mathrm{s}^{-1}\end{array}$ & $\begin{array}{c}\langle n(\mathrm{e})\rangle \\
\mathrm{cm}^{-3}\end{array}$ & $\begin{array}{c}I([\mathrm{C} \mathrm{II}]) \\
\mathrm{K} \mathrm{km} \mathrm{s}^{-1}\end{array}$ & $\begin{array}{c}\langle n(\mathrm{e})\rangle \\
\mathrm{cm}^{-3}\end{array}$ & $\begin{array}{c}I([\mathrm{~N} \mathrm{II}]) / \\
\mathrm{I}([\mathrm{C} \mathrm{II}])\end{array}$ & $\begin{array}{c}I_{\text {ion }}([\mathrm{C} \mathrm{II}]) \\
\mathrm{K} \mathrm{km} \mathrm{s}^{-1}\end{array}$ & $\begin{array}{c}I_{\text {ion }}([\mathrm{C} \text { II }]) / \\
I_{\text {tot }}([\mathrm{C} \text { II }])\end{array}$ & $\begin{array}{c}I(\mathrm{H} \mathrm{I}) \\
\mathrm{K} \mathrm{km} \mathrm{s}^{-1}\end{array}$ & $\begin{array}{c}I_{\mathrm{HI}}([\mathrm{C} \mathrm{II}])^{a} \\
\mathrm{~K} \mathrm{~km} \mathrm{~s}^{-1}\end{array}$ \\
\hline-0.25 to +0.25 & $110-125$ & 4.05 & $0.9^{b}$ & 10.8 & $0.8^{b}$ & 0.37 & 9.7 & 0.89 & 521 & 0.5 \\
0.60 to 0.90 & $110-125$ & 0.82 & $0.4^{b}$ & 2.2 & $0.3^{b}$ & 0.38 & 2.0 & 0.92 & 251 & 0.2 \\
-0.25 to +0.25 & $70-110$ & 25.9 & $40^{c}$ & 49.2 & $18^{c}$ & 0.53 & 44.9 & $<0.91^{d}$ & 3700 & 3.4 \\
\hline
\end{tabular}

Notes. ${ }^{(a)}$ The $[\mathrm{C} \mathrm{II}]$ intensity from the $\mathrm{HI}$ gas is calculated assuming $n(\mathrm{H})=20 \mathrm{~cm}^{-3} .{ }^{(b)} n(\mathrm{e})$ for the WIM is derived from $I_{\text {ion }}([\mathrm{N} \mathrm{II}])$ and $I([\mathrm{C} \mathrm{II}])$ assuming $L=1 \mathrm{kpc}{ }^{(c)} n(\mathrm{e})$ in the ionized boundary layers is derived from $I_{\text {ion }}([\mathrm{N} \mathrm{II}])$ and $I([\mathrm{C} \mathrm{II}])$ assuming $L=8$ pc. ${ }^{(d)}$ This value is only an upper limit as there is evidence for foreground and/or self-absorption of [C II] - see text.

In Table 1 we give the [C II] intensity calculated to arise from the WIM, $I_{\text {ion }}([\mathrm{C} \mathrm{II}])$, corresponding to $V_{\mathrm{LSR}}=110-125 \mathrm{~km} \mathrm{~s}^{-1}$, and the fraction of the total observed intensity, $I_{\text {tot }}([\mathrm{C}$ II $]$ ) (the molecular regions are discussed in Sect. 4.4.2). The [C II] intensity calculated to arise from the highly ionized gas, $I_{\text {ion }}([\mathrm{C} \mathrm{II}])$, from just the WIM, $V_{\mathrm{LSR}}=110-125 \mathrm{~km} \mathrm{~s}^{-1}$, ranges from $2.0 \mathrm{~K} \mathrm{~km} \mathrm{~s}^{-1}$ out of the plane $(b=0.6$ to 0.9$)$ to $9.7 \mathrm{~K} \mathrm{~km} \mathrm{~s}^{-1}$ in the plane $(b= \pm 0.25)$. In and out of the plane the average fraction $I_{\text {ion }}([\mathrm{C} \mathrm{II}]) / I_{\text {tot }}([\mathrm{C} \mathrm{II}])$ arising from the WIM is $\sim 90 \%$. Thus the $[\mathrm{C} \mathrm{II}]$ emission from the WIM is a significant fraction of the observed [C II] intensity towards the WIM, as might be expected from this highly ionized low density medium with very little neutral gas.

If $\sim 90 \%$ of the [C II] emission comes from the highly ionized WIM, then the remaining $\sim 10 \%$ probably comes from the $\mathrm{HI}$ gas clouds mixed in with the WIM, as there is no molecular gas present. In Fig. 6 we see that there is H I emission from 110 to $125 \mathrm{~km} \mathrm{~s}^{-1}$. In Table 1 we give the average $I(\mathrm{H} \mathrm{I})$ in and above the plane, which can readily be converted to an equivalent column density, $N\left(\mathrm{C}^{+}\right)=1.82 \times 10^{18} x\left(\mathrm{C}^{+}\right) I(\mathrm{HI})$. The corresponding $\mathrm{C}^{+}$column densities in and above the plane are, $N\left(\mathrm{C}^{+}\right)=2.6$ and $1.3 \times 10^{17} \mathrm{~cm}^{-2}$, respectively. This column density corresponds to a mean hydrogen density, $\langle n(\mathrm{H})\rangle=1.2$ and $0.6 \mathrm{~cm}^{-3}$, respectively. However, because the H I clouds do not fill the LOS, their local density is likely to be much higher. For purposes of estimating their contribution to $I([\mathrm{C} \mathrm{II}])$ we assume a typical kinetic temperature $T_{\mathrm{k}}=100 \mathrm{~K}$ and typical $\mathrm{H}$ I cloud densities $n(\mathrm{H})=10-20 \mathrm{~cm}^{-3}$. The H I contribution to $I([\mathrm{C} \mathrm{II}]), I_{\mathrm{HI}}([\mathrm{C} \mathrm{II}])$, even for the larger value of $n(\mathrm{H})=20 \mathrm{~cm}^{-3}$, is $\sim 4 \%$ in the plane and $\sim 10 \%$ out of the plane. This percentage contribution is consistent with the difference between total [C II] intensity and the $\sim 90 \%$ contribution of the WIM to the total [C II] intensity. 


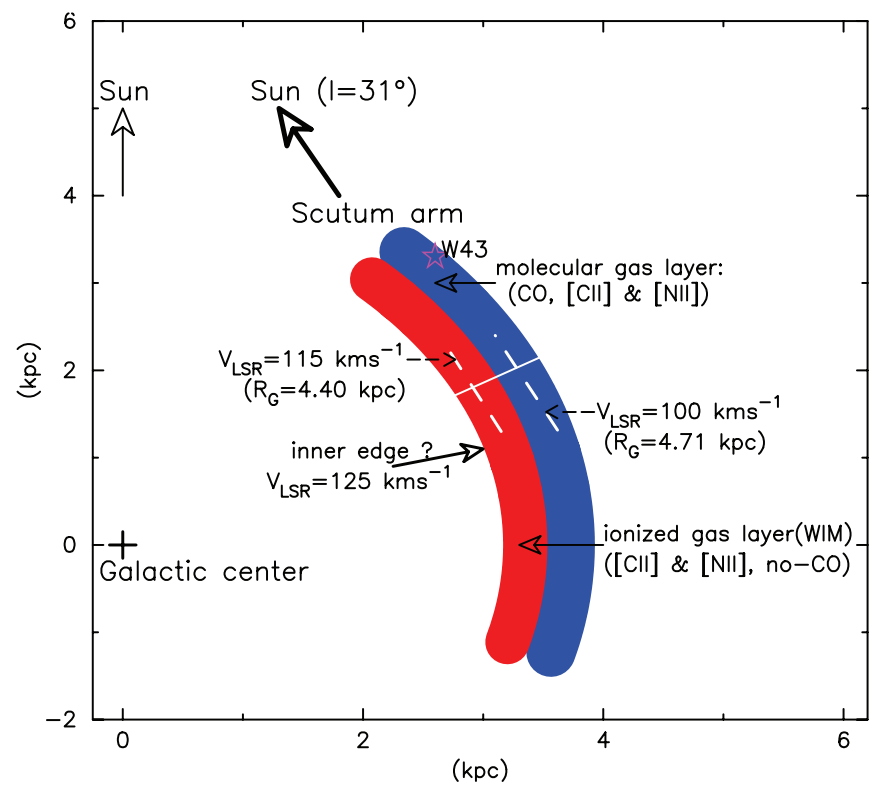

Fig. 9. A schematic radial cross section view of the spiral arm perpendicular to the LOS to Scutum spiral arm tangency showing the inner to outer edges of the spiral arm (this sketch is not to scale). The molecular gas layer traced by ${ }^{13} \mathrm{CO}$ is shown by a thick blue line. Dashed lines show two representative radial velocities and corresponding Galactic locations. The red arc indicates the spiral density wave shock. The location of W43, from the study by Zhang et al. (2014), is marked on the schematic.

\section{3. $z$-distribution}

In principle the scale height of the gas in the disk is a measure of the different forces in the disk, such as gravity and pressure, and thus the energy insertion in the disk. The mass distribution arises from stars, the ISM, and dark matter. The pressure is a result of thermal energy, random motion of clouds, cosmic ray and magnetic pressure, while supernovae and OB-associations provide energy injection that may drive gas into the halo. [C II] observations with Herschel HIFI made possible the determination of the mean distribution of the gas traced by $\mathrm{C}^{+}$. However, because [C II] traces many different gas components, to get the scale heights of the different ISM components it is necessary to separate out the different scale heights by associating the emission coming from $\mathrm{CO}, \mathrm{HI}$, and WIM regions. Velusamy \& Langer (2014) analyzed the distribution of all the [C II] emission detected by the GOT C+ survey as a function of $z$ and found that the average scale height for [C II] (all gas components) is $\sim 170 \mathrm{pc}$, larger than that for $\mathrm{CO}$, but smaller than that for $\mathrm{HI}$, as might be expected. They found that [C II] from the WIM and H I combined had a scale height $\sim 330 \mathrm{pc}$, larger than H I alone. However, with [C II] alone, they were unable to separate the scale height of the weakly and highly ionized gas. From other studies the highly ionized diffuse gas is known to have a number of components and extend high above the plane.

To determine the $z$-distribution of the ionized gas traced by [C II] and [N II] we averaged the spectra for each value of $b$ using observations at adjacent longitudes. This approach reduces the risk that we might have an unusually bright source of emission in the beam and improves the signal-to-noise at the higher latitudes where the emission is weak. To derive the $z$-distribution of the intensity of [C II] and [N II] in the WIM we restrict the calculation of intensity to $V_{\mathrm{lsr}}=110$ to $125 \mathrm{~km} \mathrm{~s}^{-1}$ where there is no $\mathrm{CO}$ gas (see Fig. 6).
In Fig. 8 we plot the integrated intensity of [C II] and [N II] as a function of $b$, for two different velocity ranges, $100-125 \mathrm{~km} \mathrm{~s}^{-1}$ (left panel) and $110-125 \mathrm{~km} \mathrm{~s}^{-1}$ (right panel), where the symbols indicate the number of averaged LOS. The left panel also includes the integrated ${ }^{13} \mathrm{CO}$ intensity profile (dashed line) as a function of $b$. The ${ }^{13} \mathrm{CO}$ intensity profile is computed using the GRS data cube (Jackson et al. 2006). Note that the CO profile near $b=0.0$ is uncertain due to complexity in the distribution of strong ${ }^{13} \mathrm{CO}$ emission as seen in Fig. 2. Nevertheless the high latitude values demonstrate how sharply the ${ }^{13} \mathrm{CO}$ emission drops. It is nearly zero by $b=0.5$, and evidently has a much smaller scale height than $[\mathrm{C}$ II] and [N II]. In the right panel, where the velocity range corresponds only to WIM gas without any evident molecular component, we find that the data points are roughly consistent with an exponential, $I=I_{0} \exp \left(-(b-\delta b) / b_{0}\right)$ with a scale height $b_{0}=0.45$ and offset from plane $\delta b=0.05$. Within the uncertainty in the measured intensities both [C II] and [N II] have similar $z$-scales of $\sim 55 \mathrm{pc}$ assuming a distance of $7 \mathrm{kpc}$ to the tangency. These scale heights do not measure the distribution of the gas density but are measures of the [C II] and [N II] emission from the WIM, because their intensities are proportional to $n(\mathrm{e})^{2}$, and thus at some point below the sensitvity of the [C II] and $[\mathrm{N}$ II] surveys.

[C II] observations with Herschel HIFI made possible the determination of the radial distribution of the gas traced by $\mathrm{C}^{+}$. However, because [C II] traces many different gas components it is necessary to separate out the different scale heights by determining the $\mathrm{C}^{+}$emission coming from $\mathrm{CO}, \mathrm{H} \mathrm{I}$, and WIM regions. To separate out the different [C II] sources, Velusamy \& Langer (2014) correlated the intensities in $3 \mathrm{~km} \mathrm{~s}^{-1}$ wide velocity bins, "spaxels", in each [C II] velocity profile with the corresponding spaxel intensities in the ${ }^{12} \mathrm{CO},{ }^{13} \mathrm{CO}$ and $\mathrm{H}$ I velocity profiles, and found that the [C II] Gaussian scale height for $z$ in the Scutum tangency is smaller than the average value for [C II] for the disk as a whole. There are a number of possible reasons for the difference. The spaxel analysis was a global average across the disk which had more sensitivity at higher values of $b$ and thus may detect more emission higher in the plane. Second, the Scutum tangency has many active regions of star formation and thus potentially more heating UV. Thus the [C II] emission in the plane $(|b| \lesssim 0.5)$ may be much stronger than the radial average in the plane found by Pineda et al. (2013) and thus skew the fit to a smaller scale height.

\subsection{The Source of [NII] and [CII] in the Scutum arm}

The schematic diagram in Fig. 9 illustrates the proposed structure of the gas layers of the Scutum arm as inferred from the observed velocity structure of the gas components traced by $[\mathrm{C} \mathrm{II}],[\mathrm{N} \mathrm{II}]$, and ${ }^{13} \mathrm{CO}$. In this picture only the WIM is traced by [N II] and [C II] at $V_{\mathrm{LSR}}>110 \mathrm{~km} \mathrm{~s}^{-1}$ and the red curve indicates where a shock might form, while the denser molecular gas layer is traced by ${ }^{13} \mathrm{CO}$ at $V_{\mathrm{LSR}}<110 \mathrm{~km} \mathrm{~s}^{-1}$. We use the unique viewing geometry (as shown in the Fig. 9) of the tangency to derive the cross-section view across the spiral arm, from the inner to outer edge, as a function Galacto-centric radial distance $\left(R_{\mathrm{G}}\right)$. We use the $V_{\mathrm{LSR}}-R_{\mathrm{G}}$ relationship assuming pure Galactic rotation, with rotation velocities of $235 \mathrm{~km} \mathrm{~s}^{-1}$ and $220 \mathrm{~km} \mathrm{~s}^{-1}$ at $R_{\mathrm{G}}=4 \mathrm{kpc}$ and $R_{\text {sun }}=8.34 \mathrm{kpc}$, respectively (cf. Reid et al. 2014, 2016). Thus the velocity resolved [C II], [N II], and ${ }^{13} \mathrm{CO}$ spectral data delineate the gas layers of the spiral arm. The low surface brightness [C II] and [N II] emission observed at $V_{\mathrm{LSR}}>110 \mathrm{~km} \mathrm{~s}^{-1}$, which are without associated ${ }^{13} \mathrm{CO}$, is clearly from low density ionized WIM gas located 
along the inner boundary of the spiral arm. On the other hand the brighter [C II] and [N II] emission seen with associated ${ }^{13} \mathrm{CO}$ at $V_{\mathrm{LSR}}<110 \mathrm{~km} \mathrm{~s}^{-1}$ is clearly from the denser ionized gas mixed with the population of dense molecular gas along the mid-layers of the spiral arm. Using the [C II] emission alone to trace ionized gas, in combination with CO, Velusamy et al. (2015) have shown in the Norma and Perseus tangencies similar evidence for the segregation of the WIM ionized gas layer being displaced inward towards the Galactic center with respect to the dense molecular gas layers of the spiral arm. In the present case of the Scutum tangency, the combination of both [C II] and [N II] tracing the ionized gas component supports our prior interpretation of the gas layers distributed from inner to outer edges of the spiral arm as shown in the schematic in Fig. 9.

[N II] is widespread and relatively strong throughout the Scutum spiral arm tangency with typical intensities $I_{\text {ion }}([\mathrm{NII}]) \sim$ (0.37-0.53) I([C II]) (Table 1 Col. 7). This ratio is much larger than $\sim 0.1$ to 0.2 found by COBE FIRAS to be typical of the Galactic distribution. The SOFIA $b$ scans show that $[\mathrm{N}$ II] is strongest at the midplane, where the dense molecular cloud tracer ${ }^{13} \mathrm{CO}$ is strongest, and decreases with latitude exponentially with a scale height of 0.57 and is essentially undetectable beyond 1.7. Thus the [N II] emission observed by COBE FIRAS, with a $7^{\circ}$ beam, would have been significantly beam diluted. Here we discuss the conditions and sources of the relatively large electron densities responsible for the [N II] emission and the large $[\mathrm{N} \mathrm{II}]$ to $[\mathrm{C}$ II] ratio in the Scutum arm and by extension in the sparse galactic surveys of [N II] and [C II] (Goldsmith et al. 2015; Langer et al. 2016).

\subsubsection{WIM component}

The electron densities in the WIM derived from [C II] emission in Velusamy et al. $(2012,2015)$ and from [N II] in this paper, are much larger than those characteristic of the Galactic interarm WIM derived using other probes. In the velocity range $V_{\mathrm{LSR}}=110-125 \mathrm{~km} \mathrm{~s}^{-1}$, where there is no evidence of molecular clouds as indicated by the absence of ${ }^{13} \mathrm{CO}$, the [N II] emission arises almost entirely from the WIM. This result is supported by our estimate that $\sim 90 \%$ of the observed [C II] intensity is associated with the $\mathrm{N}^{+}$gas in this velocity range. The WIM average electron density within $b= \pm 0.25$ is $\sim 0.4$ to $0.9 \mathrm{~cm}^{-3}$, or about 5 to 20 times larger than the average WIM density, $0.03-0.08 \mathrm{~cm}^{-3}$ (Haffner et al. 2009), that fills the bulk of the Galactic disk. We interpret this density increase in the inner edge of the arm as due to compression of the interarm WIM in the gravitational potential of the leading edge of the inner spiral arm.

\subsubsection{High density ionized gas in the Scutum molecular layer}

Unlike the WIM component observed at $V_{\mathrm{LSR}}>110 \mathrm{~km} \mathrm{~s}^{-1}$ interpreting the strong [N $\mathrm{NI}]$ and $[\mathrm{C} \mathrm{II}]$ emission features at $V_{\mathrm{LSR}}<$ $110 \mathrm{~km} \mathrm{~s}^{-1}$ is more challenging as they are mixed with the denser molecular gas component in the spiral arm. These LOSs are located in the W43 complex (W43-south; see Fig. 9), which shows evidence for shocks and compression flows (e.g. Carlhoff et al. 2013) with enhanced star formation. To analyze individual features, we ideally need the densities and/or physical size of the emitting regions. In principle, if we could observe spectrally resolved [N II] at $122 \mu \mathrm{m}$ and $205 \mu \mathrm{m}$ we could constrain the density of the ionized gas in the emitting region as a function of $V_{\text {LSR }}$. However, the high spectral resolution HIFI instrument on Herschel operated only beyond $157 \mu \mathrm{m}$ and Herschel PACS only had a resolving power of $\simeq 1000$ at $122 \mu \mathrm{m}$. Presently no heterodyne instrument is available to observe the $122 \mu \mathrm{m}$ line.

In their Galactic survey Goldsmith et al. (2015) observed [N II] with PACS at two lines of sight in the Scutum arm, $(l$, $b)=(30.0,0.0)$ and $(31.2766,0.0)$. They used the two [N II] transitions with an excitation model to derive the corresponding electron densities, $n(\mathrm{e})=29$ and $31 \mathrm{~cm}^{-3}$ and column densities, $N\left(\mathrm{~N}^{+}\right)=7.7 \times 10^{16}$ and $11.4 \times 10^{16} \mathrm{~cm}^{-2}$. They also observed $(31.2766,0.0)$ in [N II] $205 \mu \mathrm{m}$ with Herschel HIFI and the spectrum shows that nearly all the [N II] emission was coming from the Scutum arm (Fig. 1 in Langer et al. 2016). The [N II] spectrum of $(30 \% 0,0.0)$ included here (Fig. 5) also shows [N II] emission throughout the arm. Furthermore, PACS detected [N II] emission across the 25-pixel array which has a $\sim 45^{\prime \prime}$ footprint, corresponding to about $1.5 \mathrm{pc}$ at the distance to the Scutum arm. Thus it is reasonable to surmise that the Scutum arm is an extended source of dense highly ionized gas that coexists with the molecular gas, perhaps as an ionized boundary layer associated with the molecular clouds.

We can estimate the average path length of the emitting region observed with PACS, $L=N\left(\mathrm{~N}^{+}\right) x\left(\mathrm{~N}^{+}\right) / n(\mathrm{e})$, by substituting the values for $n(\mathrm{e})$ and $N\left(\mathrm{~N}^{+}\right)$from Table 2 in Goldsmith et al. (2015). We find $L=6.1 \mathrm{pc}$ and $8.5 \mathrm{pc}$ for the emitting regions in $\left(30^{\circ} 0,0 \circ 0\right)$ and $(31.2766,0.0)$, respectively. However, because the PACS data are spectrally unresolved we cannot separate how much [N II] $122 \mu \mathrm{m}$ emission comes from the WIM tangency and how much from within the molecular arm, which may include dense compact or extended H II regions or shocked gas. Thus an exact calculation of the density of the different ionized components from an excitation model is not possible without the spectrally resolved $122 \mu \mathrm{m}$ line.

Instead, to estimate the average conditions of highly ionized gas in the molecular layers of the arm, we use only the emission in the velocity range where ${ }^{13} \mathrm{CO}$ is detected, $V_{\mathrm{LSR}}=70$ to $110 \mathrm{~km} \mathrm{~s}^{-1}$. In Table 1 (row 3) we list the [C II] and [N II] intensities for this velocity range. If we assume that most of this emission comes from a region with a total length of $6-8 \mathrm{pc}$, as discussed above, then $n(\mathrm{e}) \sim 40 \mathrm{~cm}^{-3}$ from $I_{\text {ion }}([\mathrm{N} \mathrm{II}])$ and $\sim 18$ from $I([\mathrm{C}$ II $])$. Thus the densities derived here bracket the values in Goldsmith et al. (2015) and are consistent with the PACS excitation analysis. Such high densities are indicative of H II regions and the ionized boundary layers (IBL) of dense clouds (Abel et al. 2005; Abel 2006). The multiple velocity features seen in each of the HIFI and GREAT [N II] spectra show that the total path length of $8 \mathrm{pc}$ will then correspond to about 6 to $10 \mathrm{H}$ II or IBL components with an average individual depth of $\sim 1 \mathrm{pc}$.

Table 1 (row 3) lists the intensity of [C II] that arises from the $[\mathrm{N} \mathrm{II}]$ component, $I_{\text {ion }}([\mathrm{C} \mathrm{II}])=44.9 \mathrm{~K} \mathrm{~km} \mathrm{~s}^{-1}$, derived using Eq. (4). The predicted fractional [C II] intensity from the highly ionized gas, $I_{\text {ion }}([\mathrm{C}$ II $]) / I_{\text {tot }}([\mathrm{C}$ II $])=91 \%$. In addition, as seen in Table 1, the contribution of [C II] from H I clouds in the plane is $\sim 7 \%$. This result is surprising because it would indicate that very little [C II] emission comes from PDRs and $\mathrm{CO}$-dark $\mathrm{H}_{2}$ clouds, which have significant amounts of $\mathrm{C}^{+}$and much higher densities than the H I clouds. However, as seen in Fig. 5 and discussed in Sect. 3.1, there is evidence for multiple [C II] absorption features and therefore the observed $I_{\mathrm{tot}}([\mathrm{C} \mathrm{II}])$ is a lower limit to the intrinsic source intensity. Langer et al. (2016) found similarly low $[\mathrm{C}$ II] to $[\mathrm{N} \mathrm{II}]$ ratios, sometimes with $[\mathrm{N} \mathrm{II}] \geq[\mathrm{C} \mathrm{II}]$, towards several of the ten LOS they observed in [C II] and [N II] with HIFI. In several components they were able to correct for the absorption and the percentage of emission from the ionized gas ranged from $34 \%$ to $58 \%$ instead of nearly $100 \%$. Unfortunately the 
crowding of features along the LOS to the Scutum tangency, as seen in Figs. 2 and 5, makes it difficult to correct for the [C II] absorption using the approach described by Langer et al. (2016). The large fraction of [C II] arising from [N II] regions is not just a phenomenon in the Galactic plane as Röllig et al. (2016) observed $[\mathrm{C} \mathrm{II}]$ and $[\mathrm{N}$ II] with GREAT in the nearby spiral galaxy IC 342 , and showed that $30-90 \%$ of [C II] arises from the highly ionized regions traced by [N II].

That the [N II] emission is detected throughout the velocity range of the molecular gas, $V_{\mathrm{LSR}}=70$ to $110 \mathrm{~km} \mathrm{~s}^{-1}$, indicates that the ionized boundary layers are distributed throughout the arm. There are two potential sources of the dense highly ionized gas, photoionization by $\mathrm{O}$ stars and shock compression of the WIM. In the GRS survey, which includes the Scutum tangency, Anderson et al. (2009) report high, 80\%, morphological association between $\mathrm{CO}$ and $\mathrm{H}$ II regions, which could account for the wide spread [N II] associated with ${ }^{13} \mathrm{CO}$ in the SOFIA spectra. However, a more likely source of the widespread dense $n(\mathrm{e})$ in the [N II] layers is shock compression as the WIM gas falling into the gravitational potential of the arm, which can accelerate the WIM at the inner gas layer of the spiral arm to a velocity of several $\mathrm{km} \mathrm{s}^{-1}$, much greater than the sound speed in the neutral gas clouds, $\sim 1 \mathrm{~km} \mathrm{~s}^{-1}$, resulting in a shock at the interface. This mechanism would also explain the high $n(\mathrm{e})$ densities distributed along about 100 LOS throughout the inner galaxy (Goldsmith et al. 2015). In the Scutum arm we might expect to see some shocked gas with enhanced [C II] and [N II] emissions due to the proximity of the end of the Galactic bar and spiral arm interaction region. However, the WIM (at $V_{\mathrm{LSR}}>110 \mathrm{~km} \mathrm{~s}^{-1}$ ) is fully devoid of $\mathrm{CO}$, that is without any molecular gas, which is more consistent with compression of the interarm WIM by gravitational infall rather than gas streaming from the Galactic bar. Therefore, in our model the ionized boundary layers are continually fueled by the incoming stream of gas.

\section{Summary}

We report high spectral resolution observations of [C II] and [N II] in longitude and latitude across the Scutum spiral arm tangency using upGREAT and GREAT, respectively, on SOFIA. We use these tracers to characterize the highly ionized gas of the WIM in terms of its electron density, the fraction of [C II] that arises from the highly ionized gas, and the scale height of [C II] and [N II] emission. The high spectral resolution observations allow us to separate the $[\mathrm{N} \mathrm{II}]$ and $[\mathrm{C}$ II] emission from the WIM from that in the neutral arm. The WIM emission over the inner leading edge of the Scutum arm extends from $V_{\mathrm{LSR}}=110$ to $125 \mathrm{~km} \mathrm{~s}^{-1}$. An analysis of the [N II] intensity from the WIM indicates an average electron density, $n(\mathrm{e}) \sim 0.9 \mathrm{~cm}^{-3}$ within $b= \pm 0.25$, approximately one to two orders of magnitude denser than the interarm WIM. This difference is indicative of compression of the interarm WIM as it flows onto the Scutum arm. The results of combining [N II] and [C II] support unambiguously the earlier results of Velusamy et al. $(2012,2015)$ on the properties of the WIM along spiral arm tangencies based on [C II] alone. We find that the scale height for the [C II] and [N II] emission, $\sim 55 \mathrm{pc}$, extends beyond that of the molecular gas tracer ${ }^{13} \mathrm{CO}$. A comparison of the $[\mathrm{C} \mathrm{II}]$ and $[\mathrm{N}$ II] emission in the WIM shows that a significant fraction, $\sim 90 \%$ of the $[\mathrm{C} \mathrm{II}]$ arises from the highly ionized gas.

There is widespread, relatively strong [N II] emission associated with the molecular regions of the Scutum arm, implying that there is highly ionized gas within the arm with $n(\mathrm{e}) \sim 20$ $40 \mathrm{~cm}^{-3}$ in several thin clumps or layers, totaling about $8 \mathrm{pc}$ in depth. This high density $n(\mathrm{e})$ and accompanying [N II] could be associated with $\mathrm{H}$ II regions distributed throughout the arm, but is most likely the result of shock compression of the WIM, accelerated by the gravitational potential of the Scutum arm. This process may also explain the strong [N II] emission, high electron densities, and relatively large [N II] to [C II] emission observed in recent sparse [N II] surveys of the inner Galaxy (Goldsmith et al. 2015; Langer et al. 2016). In the molecular arm we can only set an upper limit on the fraction of [C II] emission that arises from the highly ionized gas traced by [N II] because of absorption of the $[\mathrm{C} \mathrm{II}]$ emission by foreground gas. More extensive maps of spectrally resolved [N II] and [C II] of the spiral arms are needed to examine the relatively strong [N II] emission in the Scutum arm and along many other lines of sight in the Galactic disk.

Acknowledgements. We thank the referee for a careful reading of the manuscript and a number of comments that improved the content. We also thank the SOFIA engineering and operations teams for their support which enabled the observations presented here. The research reported here is based largely on observations made with the NASA/DLR Stratospheric Observatory for Infrared Astronomy (SOFIA). SOFIA is jointly operated by the Universities Space Research Association, Inc. (USRA), under NASA contract NAS2-97001, and the Deutsches SOFIA Institut (DSI) under DLR contract 50 OK 0901. This work was performed at the Jet Propulsion Laboratory, California Institute of Technology, under contract with the National Aeronautics and Space Administration. USA Government sponsorship acknowledged.

\section{References}

Abel, N. P. 2006, MNRAS, 368, 1949

Abel, N. P., Ferland, G. J., Shaw, G., \& van Hoof, P. A. M. 2005, ApJS, 161, 65 Anderson, L. D., Bania, T. M., Jackson, J. M., et al. 2009, ApJS, 181, 255

Asplund, M., Grevesse, N., Sauval, A. J., \& Scott, P. 2009, ARA\&A, 47, 481

Carlhoff, P., Nguyen Luong, Q., Schilke, P., et al. 2013, A\&A, 560, A24

Cordes, J. M., \& Lazio, T. J. W. 2003, ArXiv e-prints

[arXiv: astro-ph/0301598]

Cox, D. P. 2005, ARA\&A, 43, 337

Ferrière, K. M. 2001, Rev. Mod. Phys., 73, 1031

Goldsmith, P. F., Yıldız, U. A., Langer, W. D., \& Pineda, J. L. 2015, ApJ, 814, 133

Haffner, L. M., Dettmar, R.-J., Beckman, J. E., et al. 2009, Rev. Mod. Phys., 81, 969

Haffner, L. M., Reynolds, R. J., Babler, B. L., et al. 2016, in AAS Meeting Abstracts, 227, 347.17

Heyminck, S., Graf, U. U., Güsten, R., et al. 2012, A\&A, 542, L1

Jackson, J. M., Rathborne, J. M., Shah, R. Y., et al. 2006, ApJS, 163, 145

Langer, W. D., Goldsmith, P. F., Pineda, J. L., et al. 2015, A\&A, 576, A1

Langer, W. D., Goldsmith, P. F., \& Pineda, J. L. 2016, A\&A, 590, A43

Persson, C. M., Gerin, M., Mookerjea, B., et al. 2014, A\&A, 568, A37

Pineda, J. L., Langer, W. D., Velusamy, T., \& Goldsmith, P. F. 2013, A\&A, 554, A103

Reid, M. J., Menten, K. M., Brunthaler, A., et al. 2014, ApJ, 783, 130

Reid, M. J., Dame, T. M., Menten, K. M., \& Brunthaler, A. 2016, ApJ, 823, 77

Reynolds, R. J., Sterling, N. C., Haffner, L. M., \& Tufte, S. L. 2001, ApJ, 548, L221

Risacher, C., Güsten, R., Stutzki, J., et al. 2016, A\&A, 595, A34

Röllig, M., Simon, R., Güsten, R., et al. 2016, A\&A, 591, A33

Roman-Duval, J., Jackson, J. M., Heyer, M., et al. 2009, ApJ, 699, 1153

Shaver, P. A., McGee, R. X., Newton, L. M., Danks, A. C., \& Pottasch, S. R. 1983, MNRAS, 204, 53

Stil, J. M., Taylor, A. R., Dickey, J. M., et al. 2006, AJ, 132, 1158

Tayal, S. S. 2011, ApJS, 195, 12

Vallée, J. P. 2008, ApJ, 681, 303

Velusamy, T., \& Langer, W. D. 2014, A\&A, 572, A45

Velusamy, T., Langer, W. D., Pineda, J. L., \& Goldsmith, P. F. 2012, A\&A, 541, L10

Velusamy, T., Langer, W. D., Goldsmith, P. F., \& Pineda, J. L. 2015, A\&A, 578, A135

Young, E. T., Becklin, E. E., Marcum, P. M., et al. 2012, ApJ, 749, L17

Zhang, B., Moscadelli, L., Sato, M., et al. 2014, ApJ, 781, 89 